\title{
E.U. paediatric MOG consortium consensus: Part 2 - Neuroimaging features of paediatric myelin oligodendrocyte glycoprotein antibody-associated disorders
}

Matthias Baumanna, Frederik Bartels ${ }^{b}$, Carsten Finke ${ }^{b}$, Catherine Adamsbaum ${ }^{c}$, Yael Hacohen ${ }^{d}$, Kevin Rostásy ${ }^{\mathrm{e}^{*}}$, on behalf of the E.U. paediatric MOG consortium**

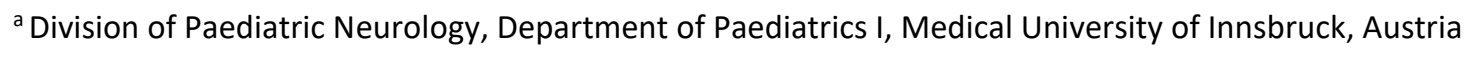

${ }^{\mathrm{b}}$ Department of Neurology, Charité - Universitätsmedizin Berlin / Berlin School of Mind and Brain, HumboldtUniversität zu Berlin, Germany

c Assistance Publique-Hôpitaux de Paris, Hôpital Bicêtre, Paediatric Radiology Department, Le Kremlin-Bicêtre, France

${ }^{d}$ Department of Neuroinflammation, Queen Square Multiple Sclerosis Centre, UCL Institute of Neurology / Department of Paediatric Neurology, Great Ormond Street Hospital for Children, London, United Kingdom e Department of Paediatric Neurology, Children's Hospital Datteln, University Witten/Herdecke, Germany

* last senior author

** E.U. paediatric MOG consortium: Arlette L. Bruijstens, Eva-Maria Wendel, Christian Lechner, Markus Breu, Lorraine Flet-Berliac, Aliénor de Chalus, Marco Capobianco, Giorgi Laetitia, Cheryl Hemingway, Evangeline Wassmer, Ming Lim, Ronny Wickström, Thaís Armangue, Kumaran Deiva, Rinze F. Neuteboom

\section{Corresponding author:}

Matthias Baumann, MD

Division of Paediatric Neurology

Department of Paediatrics I, Medical University Innsbruck

Anichstr. 35, 6020, Innsbruck, Austria

E-mail: matthias.baumann@tirol-kliniken.at

\section{Conflicts of interest}


Matthias Baumann has no conflict of interest to declare

Frederik Bartels has no conflict of interest to declare

Carsten Finke has no conflict of interest to declare

Catherine Adamsbaum has no conflict of interest to declare

Yael Hacohen has no conflict of interest to declare

Kevin Rostásy has no conflict of interest to declare relevant to this manuscript

\section{Funding sources}

This research did not receive any specific grant from funding agencies in the public, commercial, or notfor-profit sectors.

Abstract word count, 187; Text word count, 3119; References, 66

The paper contains 2 Figures and 1 Table

Keywords: MOG, MRI, Paediatrics, ADEM, Optic Neuritis, Transverse Myelitis

\section{Abbreviations}

ADEM Acute disseminated encephalomyelitis

ADEMON $\quad$ ADEM followed by optic neuritis

ADS Acquired demyelinating syndrome

AE $\quad$ Autoimmune encephalitis

AQP4 Aquaporin-4

AQP4-ON AQP4-ab-associated ON

CNS Central nervous system

DTI Diffusion tension imaging

FLAIR Fluid-attenuated inversion recovery

LETM Longitudinal extensive transverse myelitis 


$\begin{array}{ll}\text { MDEM } & \text { Multiphasic disseminated encephalomyelitis } \\ \text { MOG-abs } & \text { Myelin oligodendrocyte glycoprotein antibodies } \\ \text { MOG-ON } & \text { MOG-ab-associated ON } \\ \text { MOGAD } & \text { MOG-ab-associated disorders } \\ \text { MPRAGE } & \text { Magnetization prepared rapid acquisition gradient echo } \\ \text { MS } & \text { Multiple sclerosis } \\ \text { MS-ON } & \text { MS associated ON } \\ \text { NMOSD } & \text { Neuromyelitis optica spectrum disorders } \\ \text { ON } & \text { Optic neuritis } \\ \text { TM } & \text { Transverse myelitis }\end{array}$

\section{Abstract}

Imaging plays a crucial role in differentiating the spectrum of paediatric acquired demyelinating syndromes (ADS), which apart from myelin oligodendrocyte glycoprotein antibody associated disorders (MOGAD) includes paediatric multiple sclerosis (MS), aquaporin-4 antibody neuromyelitis optica spectrum disorders (NMOSD) and unclassified patients with both monophasic and relapsing ADS. In contrast to the imaging characteristics of children with MS, children with MOGAD present with diverse imaging patterns which correlate with the main demyelinating phenotypes as well as age at presentation. In this review we describe the common neuroradiological features of children with MOGAD such as acute disseminated encephalomyelitis, optic neuritis, transverse myelitis, AQP4 negative NMOSD. In addition, we report newly recognized presentations also associated with MOG-ab such as the 'leukodystophy-like' phenotype and autoimmune encephalitis with predominant involvement of cortical and deep gray matter structures. We further delineate the features, which may help to distinguish MOGAD from other ADS and discuss the future role of MR-imaging in regards to treatment decisions and prognosis in children with MOGAD. Finally, we propose an MRI protocol for routine examination and discuss new imaging techniques, which may help to better understand the neurobiology of MOGAD. 


\section{Introduction}

Myelin oligodendrocyte glycoprotein antibodies (MOG-abs) can be detected in children presenting with a range of paediatric acquired demyelinating syndromes (ADS) such as acute disseminated encephalomyelitis (ADEM), aquaporin-4 (AQP4) antibody negative neuromyelitis optica spectrum disorders (NMOSD), ADEM followed by optic neuritis (ADEMON), multiphasic disseminated encephalomyelitis (MDEM), and optic neuritis (ON). Importantly, MOG-abs are rarely detected in children with multiple sclerosis (MS) $(1,2)$. Several studies have addressed the clinical and prognostic relevance of MOG-abs. High-titre and transient serum MOG-abs are predominantly found in young children with ADEM (1). Children with high and persisting MOG-abs are at risk of developing further attacks, in particular episodes of ON. Until recently MOG-ab-associated disorders (MOGAD) were thought to have a more benign outcome compared to other recurrent demyelinating diseases including MS or AQP4-ab-positive NMOSD. Nevertheless, recent findings from adult cohorts indicate that patients with MOG-abs may acquire neurological deficits over time, suggesting the need for immunomodulatory treatment especially in relapsing cases (3-5).

Imaging plays a crucial role in differentiating the spectrum of ADS, which apart from MOGAD includes paediatric MS, AQP4-ab-positive NMOSD and other less well-defined subgroups. In addition, physicians caring for children with ADS must be aware of the broad differential diagnosis mimicking acute demyelinating attacks such as viral encephalitis, CNS vasculitis, CNS-isolated hemophagocytic lymphohistiocytosis, brain tumors, and mitochondrial diseases (6-10).

Therefore, it is important to delineate the spectrum of MOGAD not only on clinical and laboratory but also on radiological grounds. Compared to the imaging characteristics of children with MS, children with MOGAD present with various imaging patterns which correspond often with the respective subtype as well as age at presentation $(11,12)$. In this review we describe the common neuroradiological features of the main subtypes of MOGAD in addition to unusual presentations, delineate the features, which distinguishes MOGAD from other ADS, and discuss the future role of MR- 
imaging in aiding therapy decisions and prognosis in children with MOGAD.

\section{MR imaging}

\subsection{Brain}

\subsubsection{Lesion distribution and characteristics}

Radiological features are heterogeneous and differ depending on the clinical presentation. In paediatric MOGAD ADEM represents the largest subgroup $(40-50 \%)(1,13)$. MRI typically shows bilateral supratentorial brain lesions mostly affecting the subcortical and deep white matter and the deep grey matter. Lesions are T2-hyperintense, large ( $>2 \mathrm{~cm}$ in size), blurred and poorly demarcated (Figure 1A, 1B) $(11,14,15)$. Well-demarcated lesions, only small lesions, or involvement of just one anatomical area in younger children with ADEM are indicative of MOG-ab-negative disease (14). Children with MOG-ab-positive MDEM and NMOSD often present with similar lesion characteristics as ADEM patients, especially if they are of younger age $(16,17)$. In young patients $(<7$ years) with MDEM, these T2-hyperintense lesions can be diffuse and symmetrical, mimicking a leukodystrophy like disease process, but contrast medium enhancement and often complete resolution on follow-up scans favours their inflammatory nature (Figure 1C, 1D) (18).

\subsubsection{Differentiation of MOGAD from AQP4-ab-positive NMOSD and MS in brain}

Children with AQP4-ab-positive NMOSD, similarly to adults, have lesions following the distribution of AQP4-rich areas in the brain (19). Brain lesions are primarily found in the periventricular region of the third ventricle, in the periaqueductal grey matter, and in the dorsal brainstem adjacent to the fourth ventricle. However, lesions in these 'NMOSD-specific' regions are also present in MOG-ab-positive patients $(12,17,20,21)$. Children and adults with MOGAD show poorly demarcated lesions in the brainstem or cerebellar peduncles, typically adjacent to the fourth ventricle $(12,20,21)$. MOGAD and AQP4-ab-positive disease display different imaging characteristics then MS, but overlap in brain lesion distribution despite targeting distinct cell types (oligodendrocytes vs. astrocytes) (20). Potentially lesion quality, such as poorly demarcated lesions, frequent in MOGAD, and T1-hypointense lesions, 
more frequent in AQP4-ab-positive disease, distinguishes between the two groups $(17,20)$. In addition, in AQP4-ab-positive disease, cortical grey matter is less frequently involved than in MOGAD $(20,22$, 23).

Curvilinear lesions involving subcortical U-fibres seemed to be a distinguishing feature between MS, AQP4-ab-positive NMOSD, and MOGAD in a cohort of mostly adult patients, but curvilinear lesions appear also in young children with MOG-ab-positive ADEM (Figure 1E) $(11,24)$. Lesions in the corpus callosum, absent in certain series (25), have also been described in young patients with MOG-abpositive ADEM or MDEM (Figure $1 F)(11,15,16,26)$. In addition, periventricular lesions can also be a feature in selected children with MOGAD and do not distinguish these patients from MS or AQP4-abpositive NMOSD. However, ovoid lesions perpendicular to the major axis of corpus callosum (Dawson finger type lesions) remain characteristic of MS $(11,12)$.

In general, the presence of MOG-abs pleads against the diagnosis of MS. Nevertheless, a very small subgroup of children who are diagnosed with MS does harbour MOG-abs, which are, in general, transient and decrease to undetectable levels over time $(1,2)$. These children have a typical MS-like MRI pattern with well-demarcated lesions (Figure 1G, 1H, 1I) and short myelitis, which clearly sets them apart from non-MS cases $(11,21)$. It is important to clearly separate cases initially diagnosed as MS, which show MRI features not typical for MS and on follow-up MRI resolution of lesions, and therefore should be categorized as MOGAD (e.g. NMOSD).

In pathological studies on brain biopsies, MOG-ab-positive cases show both perivenous and confluent white matter demyelination, with an over-representation of intracortical demyelinated lesions compared to typical MS $(27,28)$. The pathology for AQP4-ab-positive NMOSD is distinct from MOGAD. In AQP4-ab-positive NMOSD a severe astrocytic damage and oligodendrocyte loss is seen, whereas in MOG-ab-associated demyelination oligodendrocytes are relatively preserved. Even large demyelinating lesions in MOGAD show relative preservation of oligodendrocytes (28). These pathological findings are in line with resolution of MRI-lesions in MOGAD and persistence of lesions or cavitation in AQP4-ab-associated disease. 


\subsubsection{Newly recognized presentations}

More recently described phenotypes of MOGAD with characteristic clinical and radiological features include a leukodystrophy-like phenotype with confluent white matter lesions and autoimmune encephalitis (AE) with MOG-abs and predominant involvement of cortical and deep gray matter structures $(13,18,21,29)$. The leukodystrophy-like phenotype occurs in particular in very young children $(13,18)$. Patients show confluent largely symmetrical white matter lesions with nodular enhancement (Figure 1J). Despite resolution of lesions, this phenotype is associated with a poor outcome $(13,18)$.

In MOG-ab-associated AE, patients fulfil the diagnostic criteria of possible AE but not the IPMSSG criteria for ADEM. Imaging shows a range of cortical (Figure 1K), and adjacent juxtacortical white matter involvement, partially combined with involvement of deep grey matter structures, or bilateral limbic areas (Figure 1L) $(13,29)$. Optic nerves, deep white matter, periventricular white matter, cerebellum, or spine are initially not involved in MOG-ab-associated AE (29). Intracranial hypertension can accompany the encephalitis phenotype in children and adults $(13,30)$. Children with MOG-encephalitis seem to have a higher risk of further relapses, and imaging in children with relapses resembles children with MDEM or NMOSD (29). Unilateral cerebral cortical encephalitis with seizures is increasingly recognized in adult patients with MOG-abs (30-32) resembling children with MOG- encephalitis. Pathological studies in patients with MOG-ab-positive cortical encephalitis show subpial demyelination (28).

MOGAD with sole localization of lesions in one region like brainstem do not fit the criteria of the abovementioned subgroups but present rare manifestations of the disease (33). Neither do children who present with isolated seizures during the first episode of MOGAD with no or only subtle MRI changes $(13,34)$. Chronic lymphocytic inflammation with pontine perivascular enhancement responsive to steroids (CLIPPERS) with detection of MOG-abs later in the course has so far only been described in one adult patient (35).

\subsection{Spinal cord}




\subsubsection{Lesion distribution and characteristics}

Spinal cord involvement is often characterized by a longitudinal extensive transverse myelitis (LETM), defined as a contiguous lesion extending over three or more vertebral segments. LETM is a feature of different subgroups of MOGAD such as ADEM and NMOSD but can also occur as isolated LETM. It mainly affects the cervical and thoracic, and less often the lumbar, and conus regions (11). The grey matter of the spinal cord is preferentially involved resulting in a characteristic T2-hyperintense line running in the superior-inferior direction on sagittal images and a distinctive $\mathrm{H}$ pattern in the axial plane (Figure $1 \mathrm{M}, 1 \mathrm{~N}$ ), although the extension of lesions can change considerably among the different spinal levels $(11,36,37)$. Short transverse myelitis $(T M)$ is rarely present in teenagers, but more frequent in adult patients $(11,38)$.

\subsubsection{Differentiation of MOGAD from AQP4-ab-positive NMOSD and MS in myelitis}

The differential diagnosis includes AQP4-ab-positive myelitis, viral myelitis and spinal cord infarction $(37,39)$. Contrast enhancement and cavitation favours AQP4-ab-positive myelitis over MOG-abpositive myelitis $(36,37)$. MOG-ab-positive myelitis usually does not go along with substance defects or spinal cord atrophy. Even MRI-negative MOG-ab-positive myelitis can occur in children and adults $(40,41)$. Multiple lesions with long and short segments and conus involvement are more frequent in MOG-ab-positive myelitis than in AQP4-ab-positive myelitis $(5,36)$. Lumbar involvement is more frequent in MOGAD compared to AQP4-ab-positive NMOSD and MS $(36,37)$. Adult patients with MOGab-positive myelitis seem to present more frequently with short myelitis than children (38). Multiple short segments are common in MS $(36,37)$.

\subsection{Optic nerves}

\subsubsection{Characteristics of MOG-ab-positive $O N$}

Optic neuritis is frequent in children with MOGAD and up to half of the patients present with bilateral ON (42). The percentage of radiological bilateral ON can be even higher than clinically recorded (43). Accordingly, in children with bilateral ON, MOG-abs are present in up to $75 \%$ (44). The majority of 
children with bilateral ON appears to have a monophasic disease course (44). A small subgroup of children with bilateral ON and MOG-abs continues to have further relapses including NMOSD, ADEM or recurrent ON $(4,44)$. In mainly adult patients with chronic relapsing inflammatory optic neuropathy (CRION), MOG-abs are frequently present and should be included in the MOGAD (4). Children with MOG-ab-positive ON usually show a longitudinally extensive ON (LEON; involving more than half of the prechiasmatic optic nerve length) with a predominantly anterior affection of the optic nerve $(42,44)$. Perineural enhancement is common (Figure 1P) $(42,44)$. Chiasma and optic tract are only infrequently involved (4, 43-46).

\subsubsection{Differentiation from $A Q P 4-a b$-positive and MS associated $O N$}

In a cohort of paediatric and adult patients with a first-episode of $\mathrm{ON}$, bilateral involvement was more common in MOG-ab-associated ON (MOG-ON) and AQP4-ab-associated ON (AQP4-ON) than MS associated ON (MS-ON) (45). Optic nerve head swelling and retrobulbar involvement was more common in MOG-ON, whereas chiasmal and bilateral optic tract involvement was more common in AQP4-ON than in MOG-ON and MS-ON. MOG-ON and AQP4-ON had longer lesion lengths than MS-ON (45). In a study including 42 children with ON (22 MS-ON, 16 MOG-ON and 4 AQP4-ON), patients with MS-ON more frequently (95\%) showed an abnormal brain MRI than patients with MOG-ON (6\%) and AQP4-ON (25\%) (47).

\subsection{Enhancement and diffusion restriction in cerebral and spinal MRI}

Gadolinium enhancement of brain and spine lesions in paediatric MOGAD is less frequent and if present often more diffuse than in MS or AQP4-ab-positive $\operatorname{NMOSD}(36,37)$. Patients with a leukodystrophylike phenotype of confluent white matter lesions may show a nodular enhancement $(13,18)$. In adult AQP4-ab-positive NMOSD 'cloud-like enhancement', multiple patchy enhancing lesions with blurred margin, is frequently seen (48). Optic nerve, and as aforementioned, perineural, enhancement is common in MOG-ON (43-45).

Areas with restricted diffusion suggestive of cytotoxic oedema is not common in paediatric MOGAD 
but may be present in younger patients with $\operatorname{ADEM}(11,49)$.

\section{Age-related clinical phenotypes and imaging patterns}

Age seems to play an important role in the presentation of the different clinical phenotypes of MOGAD and is linked to distinct imaging patterns. Younger children (9 years or less) tend to have a manifestation with poorly demarcated widespread lesions in the brain. As above-mentioned, lesions involving the corpus callosum - albeit infrequent -, and curved juxtacortical lesions involving U-fibres, may also be a feature of younger patients with MOGAD. In contrast, older children (10 years or older), who commonly present with $\mathrm{ON}$ and/or TM, more often show well-demarcated, no or only small nonspecific lesions in their cerebral MRI $(11,50)$. These differences are also seen when comparing MRI features in paediatric-onset and adult-onset MOGAD (51). Probably myelin development and compaction have a significant influence on the neuroradiological presentation. Studies of normal white matter maturation using diffusion tensor imaging show region-specific changes in mean diffusivity and fractional anisotropy from infancy to childhood $(52,53)$.

\section{MR imaging during disease course and in relapsing cases}

In a Dutch study on paediatric ADEM with unknown antibody status, new lesions and enlargement of existing lesions occurred in about $50 \%$ of MRI studies performed during clinical recovery in the first three months but was rarely seen three months after the first onset of ADEM (54). On follow-up cerebral and spinal lesions frequently resolve in MOGAD. In relapsing MOGAD, such as MDEM, new episodes are associated with new MRI lesions. However, few patients also develop new lesions while they remain asymptomatic (16). In relapsing MOGAD localization of lesions can change during the disease. In younger patients (9 years or less) the brain is more likely to be affected, whereas in patients older than 9 years optic nerves are more commonly affected (50). In patients presenting with MOGencephalitis and extensive bilateral cortical involvement, progression towards general cortical atrophy and irreversible deficits is described (13). 


\section{Differential diagnosis}

Differences in lesion location and quality between MOGAD and AQP4-ab-positive NMOSD, respectively MS, are summarized in table 1. Key imaging features in MOGAD are usually the absence of destructive lesions and resolution of lesions. Differential diagnosis includes viral encephalitis, CNS vasculitis, CNSisolated hemophagocytic lymphohistiocytosis, brain tumors, and mitochondrial diseases (figure 2) (610).

\section{Suggested MRI protocol}

\subsection{Acute attack}

Depending on the clinical picture, acute imaging will focus either on brain or spine, but imaging of the entire neuroaxis is recommended at the first presentation.

Brain MRI should be performed using a magnetic field strength of at least $1.5 \mathrm{~T}$, preferably $3.0 \mathrm{~T}$, with a slice thickness of at least $3 \mathrm{~mm}$ for $2 \mathrm{D}$ sequences. The minimum requirements for clinical routine brain MRI sequences should include (i) T2-weighted spin-echo (SE) sequences in axial plane, (ii) T2 fluidattenuated inversion recovery (FLAIR) sequences in another plane than T2-SE or (even better) in threedimensional (3D) acquisition, (iii) T1-weighted SE sequences in at least axial plane or ideally 3D (MPRAGE, Magnetization Prepared Rapid Acquisition Gradient Echo) before, and (iv) after contrast agent administration, and ( $v$ ) axial diffusion-weighted imaging. Sequences dedicated to the optic nerves, including sequences with fat saturation in frontal plane, are important in the event of a clinical picture of optic neuritis.

Spinal imaging should include sagittal and axial T2-weighted sequences, and sagittal and axial T1weighted sequences, only after gadolinium administration if combined with brain MRI. The entire spinal cord should be studied. Additional axial images of the spinal cord should be performed in areas affected by lesions.

The MAGNIMS (Magnetic Resonance Imaging in MS) network consensus guidelines on the use of MRI in MS provide similar protocols with detailed descriptions (55). 
To allow for advanced MRI analyses in research settings, additional sequences can be acquired including 3D T1-weighted MPRAGE sequences for volumetric measurements, diffusion-weighted protocols for diffusion tensor imaging analyses, echo-planar imaging sequences for the acquisition of resting state data to analyse functional connectivity alterations, and quantitative multi-parameter mapping sequences to study quantitative microstructural measures of brain tissue such as iron and myelin content (56). These advanced sequences take longer time and are therefore not part of the routine MRI protocol, but can be added for dedicated research questions.

\subsection{Follow-up}

Depending on the clinical course, follow-up imaging 3-6 months after the baseline scan is recommended. As younger children may need sedation for longer MRI protocols, a reduction of sequences may be considered to allow for shorter MRI protocols, e.g. imaging may be restricted to brain or spine. Axial T2-FLAIR/T2-weighted sequences are recommended in brain MRI, spinal imaging should at least include sagittal T2-weighted sequences.

In younger patients with resolution of symptoms and a so far monophasic course, the risks of sedation have to be weighed against the value of an additional MR-imaging study. The application of intravenous gadolinium should be restricted for the differential diagnosis at the initial episode, but not for routine follow-up studies, as evidence for cerebral gadolinium deposition exists (57). The application of intravenous gadolinium may be considered for new clinical episodes.

\section{Future directions}

MR- imaging can point to MOGAD even before results of antibody-testing are available, or uncover unusual features in patients with MOGAD such as leukodystrophy-like lesions or cortical involvement. As some of these radiological phenotypes are associated with a worse outcome, MRI may influence treatment decisions and can provide prognostic clues.

New aspects of paediatric demyelinating disorders such as MOGAD may be detected with emerging imaging techniques such as diffusion tension imaging (DTI) and volumetric analysis helping to better 
understand the pathobiology of the disease.

Investigating DTI measures (e.g. fractional anisotropy and mean diffusivity) in children with monophasic ADS, it was shown that non-lesional, normal-appearing white matter did not demonstrate age-expected maturational change (58). Importantly, while there was no suggestion of progressive loss of tissue integrity, these monophasic patients failed to 'catch-up' to normal diffusivity trajectories after the second year post-ADS, indicative of both an acute and long-lasting impact of transient demyelinating illness on non-lesional white matter integrity (58).

Volumetric studies show that in paediatric MS patients, brain volume loss is present already at first clinical presentation, and that increased disease activity is associated with more severe brain volume loss (59). Longitudinal volumetric analyses in children with autoimmune encephalitis revealed significant brain volume loss and failure of age-expected brain growth (60). Likewise, volume loss and failure of age expected brain growth was also observed in children with ADEM and other monophasic ADS presentations (61), (Bartels et al. in preparation). Both MOG-antibody positive and negative ADEM patients show brain volume loss and failure of brain growth. Patients with relapsing MOGAD (MDEM) have more pronounced brain volume loss compared to monophasic patients.

Future studies might also identify functional network alterations and changes of tissue parameters such as lipid, iron and myelin content using quantitative MRI $(62,63)$. These techniques can provide quantitative histological measures of brain tissue and allow specific monitoring of myelination longitudinally and noninvasively (64). Therefore, conventional imaging techniques, showing formation and resolution of lesions, together with advanced imaging techniques, which provide quantitative parameters for maturational changes, white matter integrity or atrophy, appear to be valuable outcome measures for future clinical trials.

\section{Conclusions}

The spectrum of MRI findings in paediatric MOGAD is wider than initially thought. Standard imaging at disease onset and follow-up will identify new aspects of this disease entity demanding adaption of the currently developed classification and search for MOG-abs in unusual demyelinating or encephalitic 
syndromes. New imaging techniques might help to better understand the neurobiology of MOGAD, age-related differences, and varying outcomes. Furthermore we want encourage the leading European institutions dealing with paediatric MOGAD to use similar MR-imaging protocols in order gain more insights in this complex disease and to establish MRI markers for future treatment trials. 
Table 1

Comparison of magnetic resonance imaging (MRI) characteristics in paediatric patients with MOG antibody associated disorders (MOGAD), AQP4 antibody positive neuromyelitis optica spectrum disorders (NMOSD), and multiple sclerosis (MS)

\begin{tabular}{|c|c|c|c|}
\hline MRI characteristics & MOGAD & AQP4-NMOSD & MS \\
\hline \multicolumn{4}{|l|}{ Brain } \\
\hline Cortical lesions & + & $(+)$ & ++ \\
\hline Juxtacortical lesions & ++ & ++ & +++ \\
\hline Periventricular lesions & ++ & ++ & +++ \\
\hline Callosal lesions & + & ++ & ++ \\
\hline Dawson finger type lesions & $(+)$ & $(+)$ & ++ \\
\hline Deep gray matter lesions & ++ & + & + \\
\hline Brainstem lesions & ++ & ++ & ++ \\
\hline Poorly demarcated lesions & +++ & ++ & + \\
\hline Well-demarcated lesions & + & ++ & +++ \\
\hline T1 hypointense lesions & + & ++ & +++ \\
\hline Lesion enhancement & + & ++ & +++ \\
\hline \multicolumn{4}{|l|}{ Spinal cord } \\
\hline Longitudinal extensive transverse myelitis & +++ & +++ & + \\
\hline Cervical involvement & +++ & +++ & +++ \\
\hline Thoracic involvement & +++ & +++ & ++ \\
\hline Lumbar involvement & ++ & + & $(+)$ \\
\hline Centrally located & ++ & + & $(+)$ \\
\hline Peripherally located & $(+)$ & + & ++ \\
\hline \multicolumn{4}{|l|}{ Optic nerves } \\
\hline Bilateral optic neuritis & +++ & ++ & + \\
\hline Longitudinally extensive optic neuritis & +++ & +++ & ++ \\
\hline Anterior optic nerve involvement & +++ & + & ++ \\
\hline Posterior optic nerve involvement & + & +++ & ++ \\
\hline Chiasmal involvement & + & ++ & + \\
\hline Optic tract involvement & $(+)$ & ++ & $(+)$ \\
\hline
\end{tabular}

(+) rare, + infrequent, ++ frequent, +++ very frequent

Data summarized from several studies $(2,11,12,20,22,25,26,36,37,43-45,51,65,66)$. 


\section{Figure legends}

\section{Figure 1 - MRI features of MOGAD}

(A, B) Cerebral MRI of a 3-year-old boy with ADEM, who presented with fever, headache, dysarthria, gait disorder and right hemiparesis, showing large and blurred lesions involving both hemispheres and thalami (axial T2). (C, D) Follow-up scans 3 month later showed complete resolution of lesions (axial T2). (E) Juxtacortical curved lesions involving U-fibres in a 4-year-old-girl with ADEM (axial FLAIR). (F) Callosal lesions in a 4-year-old boy with ADEM (sagittal T2). (G) Well-demarcated, ovoid lesions in an 11-year-old girl with MS (axial T2). (H, I) Follow-up scans seven years later reveal new lesions and lesions radiating perpendicularly from the ventricular surface (axial T2, sagittal FLAIR). (J) Extensive and confluent white matter changes in a 4-year-old boy with ADEM, finally diagnosed as ADEMON (axial T2). (K) Cerebral MRI of a 14-year-old girl with encephalitis who presented to the emergency department with severe headache, mental status changes, and seizures showing pronounced signal alterations of the cortical grey matter, particularly in the right temporal-parietal lobe (axial FLAIR). She was finally diagnosed as relapsing MOGAD. (L) Bilateral signal alterations in both hippocampi in a 16year-old previously healthy boy with severe right-sided headache (coronal FLAIR), categorized as encephalitis with limbic involvement. (M, N) Spinal MRI of a 3-year-old boy with ADEM and LETM with central grey involvement and a distinctive $\mathrm{H}$ pattern in the axial plane (sagittal and axial T2). $(\mathrm{O}, \mathrm{P})$ Orbital MRI of a 9-year-old girl with unilateral optic neuritis showing signal increase, swelling, and contrast enhancement of the right optic nerve as well as of the perioptic nerve sheath (coronal fatsuppressed T2 and coronal fat-suppressed, postcontrast T1).

\section{Figure 2 - Differential diagnosis of MOGAD (selected examples)}

(A) Cerebral MRI of a 5-year-old boy with viral encephalitis (FSME), presenting with fever, headache, nausea, vomiting, and tonic-clonic seizures. (B) MRI of a 12-year-old girl with CNS vasculitis, presenting with episodes of impaired unilateral vision and hemiparesis. Magnetic resonance angiography and CSF pleocytosis pointed to the diagnosis of CNS vasculitis. After further stroke-like episodes, she was 
started on cyclophosphamide, leading to significant improvement. (C) MRI of a 9-year-old girl with posterior reversible encephalopathy syndrome, presenting with headache, vomiting, and tonic-clonic seizures. She had nephritis with transient arterial hypertonia. (D) MRI of a 10-year-old boy with anaplastic astrocytoma. (E) So-called 'UNOs' (unidentified neurofibromatosis objects), respectively focal altered signal intensity (FASI), in a 2-year-old-girl with neurofibromatosis type 1. (F) MRI of a 5year-old boy with atypical presentation of X-linked adrenoleukodystrophy. (G) Bilateral basal ganglia lesions in a 9-month-old-boy with mitochondrial disease (M. Leigh) and complex I deficiency. (H) Cerebral MRI of a 10-year-old boy who presented with irritability, vomiting, double vision, and vertigo showing hazy bilateral lesions in the white matter and thalami. A high titre of aquaporin-4 antibodies was detected and he was diagnosed with neuromyelitis optica spectrum disorder. (A-D, axial FLAIR; E$H$, axial T2)

\section{REFERENCES}

1. Hennes EM, Baumann M, Schanda K, et al. Prognostic relevance of MOG antibodies in children with an acquired demyelinating syndrome. Neurology. 2017; 89: 900-908.

2. Hacohen Y, Absoud M, Deiva K, et al. Myelin oligodendrocyte glycoprotein antibodies are associated with a non-MS course in children. Neurol Neuroimmunol Neuroinflamm. 2015; 2: e81.

3. Cobo-Calvo A, Ruiz A, Maillart E, et al. Clinical spectrum and prognostic value of CNS MOG autoimmunity in adults: The MOGADOR study. Neurology. 2018; 90: e1858-e1869.

4. Liu H, Zhou H, Wang J, Xu Q, Wei S. Antibodies to myelin oligodendrocyte glycoprotein in chronic relapsing inflammatory optic neuropathy. Br J Ophthalmol. 2019; 103: 1423-1428.

5. Mariano R, Messina S, Kumar K, Kuker W, Leite MI, Palace J. Comparison of Clinical Outcomes of Transverse Myelitis Among Adults With Myelin Oligodendrocyte Glycoprotein Antibody vs Aquaporin-4 Antibody Disease. JAMA Netw Open. 2019; 2: e1912732.

6. Rostasy K, Bajer-Kornek B, Venkateswaran S, Hemingway C, Tardieu M. Differential diagnosis and evaluation in pediatric inflammatory demyelinating disorders. Neurology. 2016; 87: S28-37.

7. Twilt M, Benseler SM. Childhood inflammatory brain diseases: pathogenesis, diagnosis and therapy. Rheumatology (Oxford). 2014; 53: 1359-1368. 
8. Yiu EM, Laughlin S, Verhey LH, Banwell BL, Canadian Pediatric Demyelinating Disease N. Clinical and magnetic resonance imaging (MRI) distinctions between tumefactive demyelination and brain tumors in children. J Child Neurol. 2014; 29: 654-665.

9. Benson LA, Li H, Henderson LA, et al. Pediatric CNS-isolated hemophagocytic lymphohistiocytosis. Neurol Neuroimmunol Neuroinflamm. 2019; 6: e560.

10. Wells $E$, Hacohen $\mathrm{Y}$, Waldman A, et al. Neuroimmune disorders of the central nervous system in children in the molecular era. Nat Rev Neurol. 2018; 14: 433-445.

11. Baumann M, Grams A, Djurdjevic T, et al. MRI of the first event in pediatric acquired demyelinating syndromes with antibodies to myelin oligodendrocyte glycoprotein. J Neurol. 2018; 265: 845-855.

12. Waters P, Fadda G, Woodhall M, et al. Serial Anti-Myelin Oligodendrocyte Glycoprotein Antibody Analyses and Outcomes in Children With Demyelinating Syndromes. JAMA Neurol. 2019; 77(1).

13. Armangue T, Olive-Cirera G, Martinez-Hernandez E, et al. Associations of paediatric demyelinating and encephalitic syndromes with myelin oligodendrocyte glycoprotein antibodies: a multicentre observational study. Lancet Neurol. 2020; 19: 234-246.

14. Baumann M, Sahin K, Lechner C, et al. Clinical and neuroradiological differences of paediatric acute disseminating encephalomyelitis with and without antibodies to the myelin oligodendrocyte glycoprotein. $J$ Neurol Neurosurg Psychiatry. 2015; 86: 265-272.

15. Konuskan B, Yildirim M, Gocmen R, et al. Retrospective analysis of children with myelin oligodendrocyte glycoprotein antibody-related disorders. Mult Scler Relat Disord. 2018; 26: 1-7.

16. Baumann M, Hennes EM, Schanda K, et al. Children with multiphasic disseminated encephalomyelitis and antibodies to the myelin oligodendrocyte glycoprotein (MOG): Extending the spectrum of MOG antibody positive diseases. Mult Scler. 2016; 22: 1821-1829.

17. Lechner C, Baumann M, Hennes EM, et al. Antibodies to MOG and AQP4 in children with neuromyelitis optica and limited forms of the disease. J Neurol Neurosurg Psychiatry. 2016; 87: 897-905.

18. Hacohen $\mathrm{Y}$, Rossor T, Mankad K, et al. 'Leukodystrophy-like' phenotype in children with myelin oligodendrocyte glycoprotein antibody-associated disease. Dev Med Child Neurol. 2018; 60: 417-423.

19. McKeon A, Lennon VA, Lotze T, et al. CNS aquaporin-4 autoimmunity in children. Neurology. 2008; 71: 93-100.

20. Jurynczyk M, Geraldes R, Probert F, et al. Distinct brain imaging characteristics of autoantibodymediated CNS conditions and multiple sclerosis. Brain. 2017; 140: 617-627. 
21. Hacohen Y, Mankad K, Chong WK, et al. Diagnostic algorithm for relapsing acquired demyelinating syndromes in children. Neurology. 2017; 89: 269-278.

22. Absoud M, Lim MJ, Appleton R, et al. Paediatric neuromyelitis optica: clinical, MRI of the brain and prognostic features. J Neurol Neurosurg Psychiatry. 2015; 86: 470-472.

23. Salama S, Khan M, Shanechi A, Levy M, Izbudak I. MRI differences between MOG antibody disease and AQP4 NMOSD. Mult Scler. 2020: 1352458519893093.

24. Jurynczyk M, Tackley G, Kong Y, et al. Brain lesion distribution criteria distinguish MS from AQP4antibody NMOSD and MOG-antibody disease. J Neurol Neurosurg Psychiatry. 2017; 88: 132-136.

25. Fernandez-Carbonell C, Vargas-Lowy D, Musallam A, et al. Clinical and MRI phenotype of children with MOG antibodies. Mult Scler. 2016; 22: 174-184.

26. Cobo-Calvo A, Ruiz A, D'Indy H, et al. MOG antibody-related disorders: common features and uncommon presentations. J Neurol. 2017; 264: 1945-1955.

27. Hoftberger R, Guo Y, Flanagan EP, et al. The pathology of central nervous system inflammatory demyelinating disease accompanying myelin oligodendrocyte glycoprotein autoantibody. Acta Neuropathol. 2020; 139: 875-892.

28. Takai Y, Misu T, Kaneko K, et al. Myelin oligodendrocyte glycoprotein antibody-associated disease: an immunopathological study. Brain. 2020; 143: 1431-1446.

29. Wegener-Panzer A, Cleaveland R, Wendel EM, et al. Clinical and imaging features of children with autoimmune encephalitis and MOG antibodies. Neurol Neuroimmunol Neuroinflamm. 2020; 7.

30. Wang L, ZhangBao J, Zhou L, et al. Encephalitis is an important clinical component of myelin oligodendrocyte glycoprotein antibody associated demyelination: a single-center cohort study in Shanghai, China. Eur J Neurol. 2019; 26: 168-174.

31. Ogawa R, Nakashima I, Takahashi T, et al. MOG antibody-positive, benign, unilateral, cerebral cortical encephalitis with epilepsy. Neurol Neuroimmunol Neuroinflamm. 2017; 4: e322.

32. Hamid SHM, Whittam D, Saviour M, et al. Seizures and Encephalitis in Myelin Oligodendrocyte Glycoprotein IgG Disease vs Aquaporin 4 IgG Disease. JAMA Neurol. 2018; 75: 65-71.

33. Hacohen Y, Nishimoto Y, Fukami Y, et al. Paediatric brainstem encephalitis associated with glial and neuronal autoantibodies. Dev Med Child Neurol. 2016; 58: 836-841. 
34. Ramanathan S, O'Grady G L, Malone S, et al. Isolated seizures during the first episode of relapsing myelin oligodendrocyte glycoprotein antibody-associated demyelination in children. Dev Med Child Neurol. 2019; 61: 610-614.

35. Symmonds M, Waters PJ, Kuker W, Leite MI, Schulz UG. Anti-MOG antibodies with longitudinally extensive transverse myelitis preceded by CLIPPERS. Neurology. 2015; 84: 1177-1179.

36. Dubey D, Pittock SJ, Krecke KN, et al. Clinical, Radiologic, and Prognostic Features of Myelitis Associated With Myelin Oligodendrocyte Glycoprotein Autoantibody. JAMA Neurol. 2019; 76: 301-309.

37. Tantsis EM, Prelog K, Alper G, et al. Magnetic resonance imaging in enterovirus-71, myelin oligodendrocyte glycoprotein antibody, aquaporin-4 antibody, and multiple sclerosis-associated myelitis in children. Dev Med Child Neurol. 2019; 61: 1108-1116.

38. Ciron J, Cobo-Calvo A, Audoin B, et al. Frequency and characteristics of short versus longitudinally extensive myelitis in adults with MOG antibodies: A retrospective multicentric study. Mult Scler. 2019; 26: 936944.

39. Ciccarelli O, Cohen JA, Reingold SC, et al. Spinal cord involvement in multiple sclerosis and neuromyelitis optica spectrum disorders. Lancet Neurol. 2019; 18: 185-197.

40. Sechi E, Krecke KN, Pittock SJ, et al. Frequency and characteristics of MRI-negative myelitis associated with MOG autoantibodies. Mult Scler. 2020: 1352458520907900.

41. Perez CA, Garcia-Tarodo S, Troxell R. MRI-Negative Myelitis Associated With Myelin Oligodendrocyte Glycoprotein Antibody Spectrum Demyelinating Disease. Child Neurol Open. 2019; 6: 2329048X19830475.

42. Song $H$, Zhou $H$, Yang $M, X u Q$, Sun M, Wei S. Clinical characteristics and outcomes of myelin oligodendrocyte glycoprotein antibody-seropositive optic neuritis in varying age groups: A cohort study in China. J Neurol Sci. 2019; 400: 83-89.

43. Zhao Y, Tan S, Chan TCY, et al. Clinical features of demyelinating optic neuritis with seropositive myelin oligodendrocyte glycoprotein antibody in Chinese patients. Br J Ophthalmol. 2018; 102: 1372-1377.

44. Wendel EM, Baumann M, Barisic N, et al. High association of MOG-IgG antibodies in children with bilateral optic neuritis. Eur J Paediatr Neurol. 2020; S1090-3798(20)30073-8.

45. Ramanathan S, Prelog K, Barnes EH, et al. Radiological differentiation of optic neuritis with myelin oligodendrocyte glycoprotein antibodies, aquaporin-4 antibodies, and multiple sclerosis. Mult Scler. 2016; 22: 470-482.

46. Chen JJ, Flanagan EP, Jitprapaikulsan J, et al. Myelin Oligodendrocyte Glycoprotein Antibody-Positive Optic Neuritis: Clinical Characteristics, Radiologic Clues, and Outcome. Am J Ophthalmol. 2018; 195: 8-15. 
47. Eyre M, Hameed A, Wright $S$, et al. Retinal nerve fibre layer thinning is associated with worse visual outcome after optic neuritis in children with a relapsing demyelinating syndrome. Dev Med Child Neurol. 2018; 60: $1244-1250$.

48. Ito S, Mori M, Makino T, Hayakawa S, Kuwabara S. "Cloud-like enhancement" is a magnetic resonance imaging abnormality specific to neuromyelitis optica. Ann Neurol. 2009; 66: 425-428.

49. Zuccoli G, Panigrahy A, Sreedher G, et al. Vasogenic edema characterizes pediatric acute disseminated encephalomyelitis. Neuroradiology. 2014; 56: 679-684.

50. Hacohen Y, Wong YY, Lechner C, et al. Disease Course and Treatment Responses in Children With Relapsing Myelin Oligodendrocyte Glycoprotein Antibody-Associated Disease. JAMA Neurol. 2018; 75: 478-487.

51. de Mol CL, Wong Y, van Pelt ED, et al. The clinical spectrum and incidence of anti-MOG-associated acquired demyelinating syndromes in children and adults. Mult Scler. 2019; 26: 806-814.

52. Mukherjee P, Miller JH, Shimony JS, et al. Diffusion-tensor MR imaging of gray and white matter development during normal human brain maturation. AJNR Am J Neuroradiol. 2002; 23: 1445-1456.

53. Ben Bashat D, Ben Sira L, Graif M, et al. Normal white matter development from infancy to adulthood: comparing diffusion tensor and high b value diffusion weighted MR images. J Magn Reson Imaging. 2005; 21: 503-511.

54. Wong YYM, van Pelt ED, Ketelslegers IA, et al. Evolution of MRI abnormalities in paediatric acute disseminated encephalomyelitis. Eur J Paediatr Neurol. 2017; 21: 300-304.

55. Rovira A, Wattjes MP, Tintore M, et al. Evidence-based guidelines: MAGNIMS consensus guidelines on the use of MRI in multiple sclerosis-clinical implementation in the diagnostic process. Nat Rev Neurol. 2015; 11: 471-482.

56. Kremer S, Renard F, Achard S, et al. Use of Advanced Magnetic Resonance Imaging Techniques in Neuromyelitis Optica Spectrum Disorder. JAMA Neurol. 2015; 72: 815-822.

57. Gulani V, Calamante F, Shellock FG, Kanal E, Reeder SB, International Society for Magnetic Resonance in M. Gadolinium deposition in the brain: summary of evidence and recommendations. Lancet Neurol. 2017; 16: 564-570.

58. Longoni G, Brown RA, MomayyezSiahkal P, et al. White matter changes in paediatric multiple sclerosis and monophasic demyelinating disorders. Brain. 2017; 140: 1300-1315.

59. Bartels F, Nobis K, Cooper G, et al. Childhood multiple sclerosis is associated with reduced brain volumes at first clinical presentation and brain growth failure. Mult Scler. 2019; 25: 927-936. 
60. Bartels F, Krohn S, Nikolaus M, et al. Clinical and Magnetic Resonance Imaging Outcome Predictors in Pediatric Anti-N-Methyl-D-Aspartate Receptor Encephalitis. Ann Neurol. 2020; 88: 148-159.

61. Aubert-Broche B, Weier K, Longoni G, et al. Monophasic demyelination reduces brain growth in children. Neurology. 2017; 88: 1744-1750.

62. Weiskopf N, Mohammadi S, Lutti A, Callaghan MF. Advances in MRI-based computational neuroanatomy: from morphometry to in-vivo histology. Curr Opin Neurol. 2015; 28: 313-322.

63. Lutti A, Dick F, Sereno MI, Weiskopf N. Using high-resolution quantitative mapping of R1 as an index of cortical myelination. Neuroimage. 2014; 93 Pt 2: 176-188.

64. Heath F, Hurley SA, Johansen-Berg H, Sampaio-Baptista C. Advances in noninvasive myelin imaging. Dev Neurobiol. 2018; 78: 136-151.

65. Fadda G, Brown RA, Longoni G, et al. MRI and laboratory features and the performance of international criteria in the diagnosis of multiple sclerosis in children and adolescents: a prospective cohort study. Lancet Child Adolesc Health. 2018; 2: 191-204.

66. Papetti L, Figa Talamanca L, Spalice A, Vigevano F, Centonze D, Valeriani M. Predictors of Evolution Into Multiple Sclerosis After a First Acute Demyelinating Syndrome in Children and Adolescents. Front Neurol. 2018; 9: 1156. 